managed by hepatologists were more likely to receive optimal UDCA dosing and have response documented at 1 year. This has important implications on accessing newer therapies. These findings will be used to review the PBC care pathway in Wales to improve adherence to standards and improve patient care

\section{PTU-013 TRANSIENT ELASTOGRAPHY (TE): A SCREENING TOOL FOR CHRONIC LIVER DISEASE IN PRIMARY CARE (PC)}

Tom Hosack*, Shahana Shahid, Oriana Macleod, Alex Evans. Royal Berkshire NHS Foundation Trust, Reading, UK

\subsection{6/gutjnl-2019-BSGAbstracts.222}

Introduction Liver disease is the third leading cause of premature death in England and Wales and is the only major causes of death on the rise. Half of all diagnoses of chronic liver disease are made when patients present to the Emergency Department with decompensated liver disease, and this late presentation incurs a poor prognosis. There has been a push to identify asymptomatic patients with advanced liver fibrosis early to prevent progression to decompensated cirrhosis (NG50 Cirrhosis in over 16s: assessment and management. NICE 2016).

TE is increasingly used to screen for advanced fibrosis in patients with risk factors for liver disease for early detection and referral to secondary care (SC)(Harman et al, Direct targeting of risk factors significantly increases the detection of liver cirrhosis in primary care: a cross-sectional diagnostic study utilising transient elastography. BMJ Open 2015). In this study, a hepatology specialist nurse (HSN) provided an outreach service to identify those with advanced fibrosis who would benefit from referral to SC.

Methods We carried out a prospective study with high risk patients undergoing TE by a HSN in four general practices in West Berkshire.

Risk factors prompting referral for TE include type 2 diabetes mellitus, obesity and excess alcohol use. Referral to SC was advised in those with a TE score $>10 \mathrm{kPa}$. Patients were given lifestyle advice and signposted to appropriate community services (eg drug and alcohol services, eat well services, etc). Data was analysed for rate of detection of significant fibrosis and subsequent diagnosis of advanced liver disease.

Results 476 patients, over a 27 month period, with one or more risk factors were identified. 455 underwent TE. 85 $(18 \%)$ had a TE score $>10 \mathrm{kPa}$, prompting referral to SC. 72 $(16 \%)$ were seen in a hepatology clinic for further assessment and management. 5 had thrombocytopenia, and 13 had splenomegaly sonographically. 28 (6.2\%) underwent a Gastroscopy for variceal surveillance, of which 3 had portal hypertensive gastropathy and 3 had gastro-oesophageal varices. There were $13(2.9 \%)$ newly diagnosed with cirrhosis, 1 with chronic HCV infection, 1 with autoimmune hepatitis, and 1 with a neuroendocrine tumour.

Conclusions $3.5 \%$ of patients with one or more risk factors for liver disease screened with TE were found to have significant liver disease and 2.9\% were cirrhotic on further assessment. This highlights the role for TE in PC to identify high risk patients who would not have otherwise presented to SC until they had progressed to decompensated liver disease. TE is a useful screening test and the majority of patients tested could be managed with advice and lifestyle modification in the community, reserving SC resources for those with higher risk of significant liver disease.

\section{PTU-014 DOES ADHERENCE TO THE BSG ENDORSED DECOMPENSATED CIRRHOSIS CARE BUNDLE IMPROVE PATIENT OUTCOMES?}

Alexander J Johnston*, Hayley Chen, Andrew Palmer, Tim O'Sullivan, Paul Clark. Mater Public Hospital, Brisbane, Australia

\subsection{6/gutjnl-2019-BSGAbstracts.223}

Introduction A BSG-endorsed care bundle for the management of decompensated cirrhosis in the first 24 hours of admission has been implemented in the North East of England resulting in improved management of these patients; however in this study patient outcomes were not followed up and there have been no subsequent published studies of its use. ${ }^{1,2}$ In our expanding hepatology unit of a small tertiary hospital in Brisbane, Australia we reviewed inpatient management of decompensated cirrhotic patients to assess morbidity, mortality and if adherence to the care bundle improves patient outcomes.

Methods We retrospectively reviewed patient notes for hospital admissions due to decompensated cirrhosis between 1/6/16 1/6/17. Admissions were scored according to care bundle adherence and divided into tertiles. Mortality, rates of readmission and inpatient length of stay (LOS) were compared between the most adherent and least adherent groups using Fisher's exact test with $\mathrm{p}$ values $<0.05$ considered significant. Results 59 admissions were reviewed consisting of 39 patients (69\% male, median age 60 years) with a median MELD 19 Only Child-Pugh B (33/59, 56\%) and C (26/59, 44\%) patients were included. The most adherent group had numerically lower 1-year mortality $(5 / 16,31 \%)$ than the least adherent group $(7 / 15,47 \%, p=0.4725)$. Readmission rates within 1 year were reduced in the most adherent group (6/17, 35\%) compared to the least adherent group (8/16, 50\%), though not statistically significant $(p=0.4905)$. Readmission rates at 30 days and 6 months were also reduced with care bundle adherence however again were not statistically significant. There was no effect on the length of hospital stay between groups.

Abstract PTU-014 Table 1 - Table showing 1-year mortality, readmission and LOS between groups

\begin{tabular}{llll}
\hline & All admissions & Least adherent & Most adherent \\
\hline Total admissions & 59 & 16 & 17 \\
Patients & 39 & 15 & 16 \\
1-year mortality & $11(28 \%)$ & $7(47 \%)$ & $5(31 \%)$ \\
Readmission within 30 days & $10(17 \%)$ & $3(19 \%)$ & $2(12 \%)$ \\
Readmission within 6 months & $26(44 \%)$ & $6(38 \%)$ & $5(29 \%)$ \\
Readmission within 1 year & $29(49 \%)$ & $8(50 \%)$ & $6(35 \%)$ \\
Median LOS, days (range) & $4(1-54)$ & $4(1-23)$ & $4(2-20)$ \\
Median MELD (range) & $19(8-30)$ & $19(10-30)$ & $19(10-30)$ \\
Median creatinine (range) & $68(35-627)$ & $75(40-273)$ & $65(46-627)$ \\
\hline
\end{tabular}

Conclusions Our results suggest reduced mortality and hospital readmission in patients more closely adherent to the BSG care bundle, however this difference did not reach statistical significance. Reasons for this include a small sample size which limited statistical power and that overall adherence to the care 